\section{Eficacia de la Azitromicina como terapia adjunta en el tratamiento de periodontitis crónica}

\author{
Azitromicin efficacy as associated therapy for chronic \\ periodontitis treatment
}

\section{Resumen}

El objetivo de la investigación fue comparar la eficacia de la administración sistémica de azitromicina asociada al raspaje y alisado radicular con la administración de enjuagatorios con clorhexidina asociados al raspaje y alisado radicular y con el tratamiento con sólo raspaje y alisado radicular, para reducir la profundidad de sondaje periodontal y mejorar los niveles de adherencia clínica en pacientes adultos con periodontitis crónica. Participaron 42 pacientes con periodontitis crónica, distribuidos aleatoriamente en tres grupos, siendo grupo A: azitromicina sistémica asociada a raspaje y alisado radicular; B: enjuagatorios con clorhexidina asociados al raspaje y alisado radicular; C: sólo raspaje y alisado radicular. Se evaluó la profundidad de sondaje periodontal y el nivel de adherencia clínica, antes de iniciar el tratamiento y luego cada mes durante los 6 meses posteriores. Los resultados obtenidos mostraron una mayor reducción en la profundidad de sondaje periodontal (Pretratamiento: $5.70 \pm 0.42 \mathrm{~mm}$; Postratamientos: 1 mes: $2.20 \pm 0.36 \mathrm{~mm}, 2$ meses: $2.14 \pm 0.33 \mathrm{~mm}, 3$ meses: $2.09 \pm 0.32 \mathrm{~mm}, 4$ meses: $2.04 \pm$ $0.29 \mathrm{~mm}, 5$ meses: $1.96 \pm 0.27 \mathrm{~mm}, 6$ meses: $1.93 \pm 0.25 \mathrm{~mm}$ ) y mejor nivel de adherencia clínica en el grupo de la administración sistémica de azitromicina en comparación a los otros grupos de tratamiento. Se demostró que la administración sistémica de azitromicina asociada al raspaje y alisado radicular fue superior a los otros tratamientos evaluados para la periodontitis crónica.

Palabras Claves: Azitromicina; Clorhexidina; Periodontitis crónica.

\section{Abstract}

The aim of this research was to compare systemic administration of azitromicin associated to root scaling and planning to the use of chlorhexidine mouth rinses associated to scaling and root planning, and scaling and root planning only for reducing probing depth and clinical attachment in adult patients with diagnosed of chronic periodontitis. For the trial 42 individuals with chronic periodontitis were selected, they were randomly distributed in three groups as follows: group A associated therapy of azitromicin with root scaling and planning; group B associated therapy with chlorhexidine and root scaling and planning; and group $\mathrm{C}$ root scaling and planning only. Probing depth and clinical attachment were recorded before treatment and every month for the next 6 months. The results showed better reduction in probing depth (before treatment: $5.70 \pm 0.42 \mathrm{~mm}$; during treatment: $1 \mathrm{st}$ month: $2.20 \pm 0.36 \mathrm{~mm}$, 2nd month: $2.14 \pm 0.33 \mathrm{~mm}$, 3rd month: $2.09 \pm 0.32 \mathrm{~mm}$, 4th month: $2.04 \pm 0.29 \mathrm{~mm}$, 5th month: $1.96 \pm$ $0.27 \mathrm{~mm}, 6$ th month: $1.93 \pm 0.25 \mathrm{~mm}$ ) and better clinical attachment in group A compared to group B and C. It was shown that systemic administration of azitromicin associated to root scaling and planning resulted in better outcomes compared to the other two treatments, when used for chronic periodontitis. Key words: Azitromicin; Chlorhexidine; Chronic periodontitis.

\section{Artículo Original}

Víctor Chumpitaz-Cerrate ${ }^{1}$, Cristian Sayán-Sánchez², Yuri Castro-Rodríguez², Eliberto Ruiz-Ramírez², César FrancoQuino², Elías Aguirre-Siancas², Cecilia Rodríguez-Vargas ${ }^{3}$, Franco TauquinoAlvarez ${ }^{4}$, Juan Eche-Herrera², Vilma Chuquihuaccha-Granda ${ }^{1}$

1. Departamento Académico de Ciencias Básicas 2. Escuela Académico Profesional de Odontología

3. Departamento Académico de Estomatología Preventiva y Social

4. Departamento Académico de Estomatología Rehabilitadora

1-4. Facultad de Odontología de la Universidad Nacional Mayor de San Marcos, Perú.

\section{Correspondencia}

Dr. CD. Victor Manuel Chumpitaz Cerrate

Laboratorio de Fisiología y Farmacología, Facultad de Odontología de la Universidad Nacional Mayor de San Marcos, Av. German Amezaga s/n, Lima 1. Perú.

Correo electrónico: vchumpitazc@unmsm.edu.pe

Coautores:

Sayán-Sánchez: criss_0975@hotmail.com Castro-Rodríguez: yuricastro_16@hotmail.com Ruiz-Ramírez: beto_4558@hotmail.com Franco-Quino: c.francoq@hotmail.com Aguirre-Siancas: eeas2003@hotmail.com Rodríguez-Vargas:

mceciliarodriguezv@gmail.com

Tauquino-Alvarez:francotauquino@hotmail.com Eche-Herrera: jjf_80@hotmail.com

Chuquihuaccha-Granda:vilmach1@hotmail.com

Fecha de recepción: 05-03-14

Fecha de aceptación: 31-10-14

\section{Introducción}

En la enfermedad periodontal se reconoce el papel fundamental de los microorganismos periodonto patógenos y su capacidad de colonizar regiones profundas del cemento radicular, túbulos dentinarios expuestos y hueso alveolar, donde no podrán ser removidos adecuadamente por el raspaje y alisado radicular. La administración sistémica de antibióticos puede ser necesaria para algunas formas de periodontitis (como la periodontitis crónica) con la finalidad de erradicar efectivamente los microorganismos periodonto patógenos, evitándose futu- ras recidivas. Los principales objetivos de la terapia periodontal son reducir la profundidad de sondaje, el sangrado y supuración al sondaje y mejorar el nivel de adherencia. ${ }^{1}$ El raspaje y alisado radicular es la terapia más utilizada, sin embargo, usualmente no consigue los cambios microbiológicos necesarios para mantener la estabilidad de los beneficios clínicos alcanzados inicialmente ${ }^{2,3}$.

Se ha utilizado exitosamente la administración de antibióticos sistémicos de forma conjunta al tratamiento mecáni$\mathrm{co}^{3}$. Su efectividad ha sido estudiada empleando tetraciclinas, amoxicilina, me- tronidazol, y combinaciones de éstos ${ }^{4}$. La administración sistémica consigue una adecuada distribución del antibiótico desde el plasma hacia los tejidos periodontales y bolsas periodontales. También erradica y previene la infección por patógenos periodontales que invaden los tejidos subepiteliales o que colonizan áreas extra dentales. Diversos estudios muestran mejora clínica luego del uso sistémico de antibióticos, sin embargo algunos resultados han sido controversiales $^{5,6,7}$.

La azitromicina posee amplio espectro contra microorganismos aerobios y 
anaerobios gramnegativos. La azitromicina se encuentra en altas concentraciones en fibroblastos y fagocitos y es distribuida hacia las áreas de inflamación como resultado del efecto quimiotáctico de los fagocitos, inhibe la expresión de citoquinas pro inflamatorias IL-1 $\beta$, IL-6 e IL-8 ${ }^{5,8}$. Estudios in vitro han demostrado que es eficaz contra Aggregatibacter actinomicetencomitans y Porphyromonas gingivalis. Estudios clínicos han demostrado altas concentraciones de azitromicina en tejidos periodonta$\operatorname{les}^{9,10}$.

El objetivo de la presente investigación fue comparar la eficacia de la administración sistémica de azitromicina asociada al raspaje y alisado radicular con la administración de enjuagatorios con clorhexidina asociados al raspaje y alisado radicular y con el tratamiento de sólo raspaje y alisado radicular, para reducir la profundidad de sondaje periodontal y mejorar el nivel de adherencia clínica en adultos con periodontitis crónica.

\section{Materiales y método}

Participaron 42 pacientes adultos del Servicio de Periodoncia del Departamento de Estomatología del Centro Médico Naval, entre varones y mujeres, de 30 a 60 años, ASA I, con diagnóstico de periodontitis crónica según el siguiente criterio: poseer por lo menos 15 piezas dentarias y al menos 6 lugares con profundidad de sondaje de 5-7 mm y un nivel de adherencia clínica de 5-10 $\mathrm{mm}$ antes de iniciar el tratamiento ${ }^{1}$. No participaron pacientes fumadores, $\mathrm{ni}$ gestantes; tampoco los que recibieron terapia con antibióticos, analgésicos o antiinflamatorios en los 30 días previos a su incorporación al estudio.

Todos los pacientes firmaron un consentimiento informado. Los pacientes se distribuyeron aleatoriamente en 3 grupos, designados como A, B y C, de 14 integrantes cada uno. En la $1^{\text {ra }}$ cita, a todos los pacientes se les realizó su historia clínica periodontal, donde se evaluaron los siguientes parámetros, que fueron registrados en el periodontograma:

a) Profundidad de sondaje periodontal (PS): con una sonda periodontal milimetrada (Hu-Friedy) se midió la distancia desde el margen gingival hasta el fondo del saco periodontal.

b) Nivel de adherencia clínica (NAC): con una sonda periodontal milimetrada
(Hu-Friedy) se midió la distancia desde la unión cemento-esmalte hasta el fondo del saco periodontal.

A continuación se inició el tratamiento con la fisioterapia y se les programó una $2^{\text {da }}$ cita en 7 días, donde a todos los pacientes se les continuó el tratamiento con la realización del raspaje y alisado radicular (RAR), primero con scaler ultrasónico (Cavitron) y luego con instrumental manual consistente en raspadores y curetas Gracey (Hu Friedy). A los pacientes del grupo A se les indicó azitromicina $500 \mathrm{mg}$ ( $\mathrm{Zi}$ $\operatorname{tax})^{\oplus}$ por vía oral cada 24 horas durante 3 días, a los del grupo $\mathrm{B}$ se les indicó enjuagatorios con $15 \mathrm{~mL}$ de clorhexidina al $0.12 \%$ (Perio Aid Tratamiento ${ }^{\circledR}$ dos veces al día durante 7 días, y a los del grupo $\mathrm{C}$ no se les indicó ninguna terapia adicional al raspaje y alisado radicular.

Las citas postratamiento fueron programadas luego de un mes posterior al RAR y luego cada mes durante los 6 meses posteriores. En cada cita, a todos los pacientes se les evaluó nuevamente la PS y NAC, y se registraron los datos en el periodontograma.

Los datos obtenidos fueron procesados y mostrados a través de un análisis descriptivo como media +/- desviación estándar. Los valores obtenidos de PS y NAC fueron comparados mediante el ANOVA de un factor. La comparación de la eficacia de los tratamientos fue realizada mediante el Análisis de Varianza con medidas repetidas (Modelo Lineal General). Se consideró un nivel de significancia para $p<0,05$.

\section{Resultados}

En la evaluación realizada antes de iniciar el tratamiento periodontal, se determinó que ninguno de los tres grupos presentó diferencias significativas entre sí $(p>0,05)$, por lo tanto se confirmó la distribución aleatoria entre los grupos.

Se observó que al primer mes postratamiento, la PS y el NAC presentan diferencias significativas entre los grupos $(p<0,05)$, siendo el grupo A el que redujo significativamente la PS y mejoró el NAC (figuras 1 y 2). En las evaluaciones realizadas en los meses 2, 3, 4, 5 y 6 , se evidenciaron diferencias significativas entre los tres grupos, siendo el grupo A el que mantuvo mejores resultados de PS y NAC, en relación a $\mathrm{B}$ y al C (figuras 1 y 2).
Figura 1. Comparación de la Profundidad de Sondaje (PS) entre grupos de tratamiento

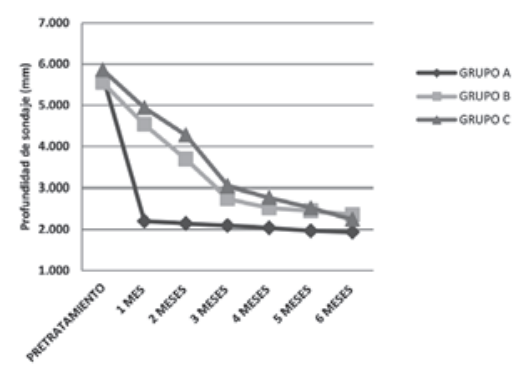

Figura 2. Comparación del Nivel de Adherencia Clínica (NAC) entre grupos de tratamiento

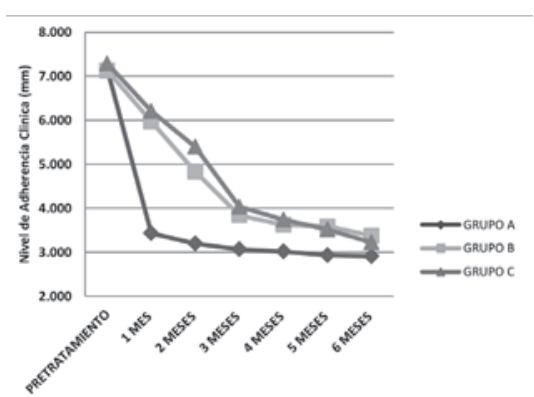

Al realizar la prueba de ANOVA de un factor y la prueba de contraste de Tukey se determinó que en relación a la PS y el NAC se puede concluir que el tratamiento A reduce más los niveles de PS y mejora los NAC en comparación al grupo $\mathrm{B}$ y $\mathrm{C}$, donde no se encontraron diferencias entre éstos dos últimos grupos (tabla 1).

$\mathrm{Al}$ evaluar la PS y el NAC en relación al tiempo y a los diferentes tratamientos, se realizó la prueba de análisis de varianza con medidas repetidas (Modelo Lineal General - MLG) existiendo diferencias significativas $(p<0,05)$ entre los grupos estudiados. Esta diferencia a favor del grupo de azitromicina se pudo evidenciar en las evaluaciones realizadas al $1^{\mathrm{er}}$ y $2^{\text {do }}$ mes, luego al $3^{\text {ro }}, 4^{\text {to }}, 5^{\text {to }}$ y $6^{\text {to }}$ mes los resultados comienzan a estabilizarse.

\section{Discusión}

La presente investigación coincide con reportes previos que indican que la administración sistémica de antibióticos produce una mejoría clínica en pacientes con periodontitis crónica. Los antibióticos utilizados incluyen tetraciclinas, penicilinas y metronidazol, agentes que deben administrarse por vía oral tres o cuatro veces al día, y por períodos de 7 a 14 días para ser efectivos ${ }^{11,12,13,14}$. Este esquema posológico puede influir de forma negativa en el cumplimiento de la terapia por los pacientes, lo cual a su vez puede ocasionar que la respuesta al tratamiento no sea la esperada ${ }^{15}$. 
Tabla 1. Estadísticos descriptivos de la profundidad de sondaje (ps) y el nivel de adherencia clínica (nac) de los grupos de tratamiento

\begin{tabular}{|c|c|c|c|c|c|c|c|c|}
\hline & \multirow[b]{2}{*}{$\begin{array}{l}\text { TIPO DE TRATA- } \\
\text { MIENTO }\end{array}$} & \multirow[b]{2}{*}{$\mathrm{N}$} & \multicolumn{3}{|c|}{ PROFUNDIDAD DE SONDAJE } & \multicolumn{3}{|c|}{ NIVEL DE ADHERENCIA CLÍNICA } \\
\hline & & & $\begin{array}{l}\text { Media } \\
(\mathrm{mm})\end{array}$ & $\begin{array}{l}\text { Desviación } \\
\text { típica }\end{array}$ & $P$ & $\begin{array}{l}\text { Media } \\
(\mathrm{mm})\end{array}$ & $\begin{array}{l}\text { Desviación } \\
\text { típica }\end{array}$ & $\mathrm{p}$ \\
\hline \multirow{2}{*}{ PRE } & GRUPOA & 14 & 5,707 & ,4160 & 0,200 & 7,157 &, 5125 & 0,200 \\
\hline & GRUPO B & 14 & 5,550 & ,3205 & & 7,129 &, 5553 & \\
\hline TRATAMIENTO & GRUPO C & 14 & 5,857 & ,4569 & & 7,286 &, 5419 & \\
\hline \multirow{3}{*}{$1 \mathrm{MES}$} & GRUPOA & 14 & 2,200 & ,3637 & 0,000 & 3,429 & ,4890 & 0,000 \\
\hline & GRUPO B & 14 & 4,529 & ,3074 & & 5,971 &, 5180 & \\
\hline & GRUPO C & 14 & 4,936 & ,3225 & & 6,207 & ,3912 & \\
\hline \multirow{3}{*}{2 MESES } & GRUPOA & 14 & 2,143 & ,3298 & 0,000 & 3,193 & ,4215 & 0,000 \\
\hline & GRUPO B & 14 & 3,686 & ,4276 & & 4,829 &, 6219 & \\
\hline & GRUPO C & 14 & 4,271 & 3970 & & 5,393 & ,3852 & \\
\hline \multirow{3}{*}{3 MESES } & GRUPOA & 14 & 2,093 & ,3198 & 0,000 & 3,064 & ,4413 & 0,000 \\
\hline & GRUPO B & 14 & 2,743 & ,5880 & & 3,836 &, 6812 & \\
\hline & GRUPO C & 14 & 3,043 & ,4536 & & 4,029 & ,4999 & \\
\hline \multirow{3}{*}{4 MESES } & GRUPO A & 14 & 2,036 & ,2898 & 0,000 & 3,014 & ,4504 & 0,002 \\
\hline & GRUPO B & 14 & 2,521 & ,4839 & & 3,621 &, 6066 & \\
\hline & GRUPO C & 14 & 2,771 & 4665 & & 3,736 &, 5486 & \\
\hline \multirow{3}{*}{5 MESES } & GRUPOA & 14 & 1,964 & ,2678 & 0,001 & 2,929 & ,3852 & 0,001 \\
\hline & GRUPO B & 14 & 2,450 & ,5080 & & 3,586 &, 5517 & \\
\hline & GRUPO C & 14 & 2,521 & 3683 & & 3,514 & ,4786 & \\
\hline \multirow{3}{*}{6 MESES } & GRUPO A & 14 & 1,936 & ,2530 & 0,016 & 2,907 &, 3647 & 0,046 \\
\hline & GRUPO B & 14 & 2,357 & ,4815 & & 3,371 &, 5703 & \\
\hline & GRUPO C & 14 & 2,243 & ,3736 & & 3,221 &, 5041 & \\
\hline
\end{tabular}

La inhibición de los microorganismos periodontopatógenos anaerobios por un período adecuado de tiempo permite un mejor entorno para una adecuada regeneración tisular y produce un cambio de composición de la flora asociada a la enfermedad periodontal hacia una flora más compatible con la salud periodontal. El metronidazol ha sido indicado para el tratamiento de infecciones periodontales anaerobias, pero el cumplimiento del paciente puede ser sólo $60 \%$ por los efectos adversos y porque requiere varias administraciones al día. La azitromicina es efectiva contra anaerobios periodontales y se administra una sola vez al día durante tres días, un esquema que favorece el cumplimiento del paciente p. $^{14}$.

La selección de la dosificación de azitromicina fue controversial, debido a que las dosis aprobadas en Estados Unidos (régimen de 5 días: primera dosis de $500 \mathrm{mg}$ y luego cuatro dosis de $250 \mathrm{mg}$ al día) y en Europa (régimen de 3 días: $500 \mathrm{mg}$ al día) son diferentes. Ambos regímenes han demostrado beneficios clínicos cuando se comparan con terapias de 10 días con otros antibióticos, mientras que el régimen de 3 días ha mostrado mejores resultados microbiológicos cuando se compara con el de 5 días. En el presente estudio se eligió el régimen de 3 días, el cual ha sido evaluado en diversos estudios ${ }^{16-20}$.

La azitromicina posee importantes características farmacocinéticas, es rápidamente absorbida hacia la sangre y posee excelente distribución en diversos tejidos incluyendo el periodonto, donde alcanza concentraciones $10-100$ veces superiores a las plasmáticas, especialmente en tejidos inflamados. Cuando se administra sistémicamente la azitromicina se concentra en tejidos periodontales permaneciendo hasta 14 días $^{14,21}$. Estos niveles tisulares exceden los niveles de MIC para Porphyromona gingivalis y Bacteroides spp. y alcanza concentraciones terapéuticas contra espiroquetas. La azitromicina es secuestrada por los leucocitos y se encuentra en altas concentraciones en el sitio de infección, desde donde es liberada con la lisis de estas células. Los fibroblastos actúan como reservorios de azitromicina y la liberan lentamente al foco de infección ${ }^{15}$.

El digluconato de clorhexidina se considera el gold standard de los antisépti- cos orales, debido a su sustantividad y adhesión a las superficies cutáneas y $\operatorname{mucosas}^{22,23}$. El chip de clorhexidina es un sistema de liberación controlada muy utilizado debido a que su biodegradación en la bolsa periodontal se da en 7-10 días, manteniendo en el fluido crevicular una MIC99 para los microorganismos aislados de la bolsa periodontal, siendo más efectivo en la reducción de la profundidad al sondaje y el mantenimiento de los niveles de inserción comparado con sólo alisado radicular. No obstante, otros estudios muestran resultados comparables utilizando sólo alisado radicular y utilizando alisado radicular adjunto al chip de clorhexidina, debido a una rápida biodegradación del sistema después de la inserción en la bolsa periodontal ${ }^{24}$.

En un estudio se evaluó el efecto de la administración sistémica de amoxicilina $500 \mathrm{mg}+$ metronidazol $250 \mathrm{mg}$, 3 veces al día durante 7 días y enjuagatorios con clorhexidina $0.12 \%$, dos veces al día durante 30 días, luego del raspaje y alisado radicular en pacientes con periodontitis agresiva. Se evaluaron: sangrado al sondaje, profundidad de sondaje y nivel de adherencia clínica. Los da- 
tos se recolectaron antes de iniciar el tratamiento, luego de 3 y 6 meses del tratamiento. A los 6 meses se observó una tendencia a incrementar los niveles de bacterias en la placa, lo que sugiere que se estaba produciendo una recolonización subgingival. Se concluyó, que la administración sistémica de amoxicilina + metronidazol y la administración tópica de clorhexidina, no redujeron el porcentaje de invasión bacteriana a las células epiteliales. Estos datos soportan la hipótesis que los reservorios bacterianos extracreviculares pueden contribuir a enfermedad periodontal recurrente $o$ refractaria en algunos pacientes ${ }^{25}$.

\section{Conclusiones}

La administración sistémica de azitromicina asociada al raspaje y alisado radicular es significativamente superior para reducir rápidamente la profundidad de sondaje periodontal y mejorar el nivel de adherencia clínica en pacientes adultos con periodontitis crónica, en comparación a la administración de enjuagatorios con clorhexidina asociados al raspaje y alisado radicular y al tratamiento con sólo raspaje y alisado radicular.

\section{Referencias bibliográficas}

1. Matarazzo F. et al. Clinical and microbiological benefits of systemic metronidazole and amoxicillin in the treatment of smokers with chronic periodontitis:a randomized placebo-controlled study. J Clin Periodontol 2008; Vol. 35: 885-896.

2. Teles RP, Haffajee AD y Socransky SS. Microbiological goals of periodontal therapy. Periodontology 2000; Vol. 42: 180-218.

3. Carvalho LH, et al. Scarlind and root planing systemic metronidazole and professional plaque removal en the treatment of chronic periodontitis in a Brazilian population II. Microbiological results. J Clin Periodontol 2005; Vol. 32: 406 -411.

4. Griffiths GS, et al. Amoxicillin and metronidazole as an adjunctive treatment in generalized aggressive periodontitis at initial therapy or re-treatment: a randomized controlled clinical trial. J Clin Periodontol 2010. Article in press.

5. Haas AN, et al. Azithromycin as an adjunctive treatment of aggressive periodontitis: 12-months randomized clinical trial. J Clin Periodontol 2008; Vol. 35: 696704.

6. Bidault P, Chandad F y Grenier D. Systemic Antibiotic therapy in the treatmeant of Periodontitis. J Can Dent Assoc. 2007; Vol. 73: 515520.

7. Heilz-Mayfield LJA. Systemic antibiotics in periodontal therapy. Aust Dent J 2009; Vol. 54: 96101.

8. Haffajee AD, Torresyap G y Socransky SS. Clinical changes following four different periodontal therapies for the treatment of chronic periodontitis: 1-year results.. .J Clin Periodontol 2007; Vol. 34: 243-253.

9. Addy LD y Martin MV. Azithromycin in dentistry - a useful agent.Br Dent J 2004; Vol. 197: 141-143.

10. Oteo A, et al. Azithromycin as an adjunct to scaling and root planing in the treatment of Porphyromonas gingivalis-associated periodontitis: a pilot study. J Clin Periodontol 2010; Vol. 37: 1005-1015.

11. Gomi K, et al. Drug concentration in inflamed periodontal tissues after systemically administered azithromycin. J Clin Periodontol 2007a; Vol. 78: 918-923.

12. Gomi K, et al. Effects of fullmouth scaling and root planing in conjunction with systemically administered azithromycin. 2007b, Journal of Periodontology, Vol. 78: 422-429.

13. Pradeep AR y Kathariya R. Clarithromycin, as an adjunct to non surgical periodontal therapy for chronic periodontitis: A double blinded, placebo controlled, randomized clinical trial. Arch Oral Biol 2011. Article in press.

14. Hirsch R. Periodontal healing and bone regeneration in response to azithromycin. Aust Dent J 2010; Vol. 55: 193-199.

15. Schmidt EF y Bretz WA. Benefits of additional courses of systemic azithromycin in periodontal disease. N Y State Dent J 2007, págs. 40-45.

16. Smith SR, et al. A doubleblind placebo-controlled trial of azithromycin as an adjunct to non-surgical treatment of periodontitis in adults: clinical results. J Clin Periodontol 2002; Vol. 29: 54-61.

17. Dastor SF, et al. Effect of adjunctive systemic azithromycin with periodontal surgery in the treatment of chronic periodontitis in smokers: a pilot study. Journal of Periodontology 2007 ; Vol. 78: 1887-1896.

18. Yashima A, et al. One-stage fullmouth versus partial-mouth scaling and root planing during the effective half-life of systemically administered azithromycin. Journal of Periodontology 2009; Vol. 80: 1406-1413.

19. Lai PCV, et al. Azithromycin concentrations in blood and gingival crevicular fluid after systemic administration. Journal of Periodontology 2011;82:15826.

20. Ho W, et al. Azythromycin decreases crevicular fluid volume and mediator content. Journal of Dental Research 2010; 89:831-5.

21. Kusofuka Y. Effect of azithromycin on anticoagulation status in warfarinized patients. Oral Surg Oral Med Oral Pathol 2013; 115:148-51.

22. Mascarenhas P, et al. Clinical response of azithromycin as an adjunct to non-surgical periodontal therapy in smokers. 2005, Journal of Periodontology, Vol. 76: 426-436.

23. Stratul SL, et al. Effect of smoking on the results of a chlorhexidine digluconate treatment extended up to 3 months after scaling and root planing-A pilot study. Quintessence Int 2011; Vol. 42: 555-563.

24. Palacio PA, Herrera LM y Duque A. Sistemas de liberación controlada de antimicrobianos en periodoncia: Revisión de la literatura. 2. Revista CES Odontología 2008; Vol. 21: 6967.

25. Johnson JD, et al. Persistence of extracrevicular bacteria reservoirs after treatment of aggressive periodontitis. 2. Journal of Periodontology 2008; Vol. 79: 2305-2312. 